\title{
Analysis and Applications of the Measurement Uncertainty in Electrical Testing
}

\author{
Hung-Lu Wang \\ Bureau of Standards, Metrology \& Inspection, MOEA, Taiwan \\ Email: hl.wang@bsmi.gov.tw
}

Received February 2015

\begin{abstract}
Recently, uncertainty measurement is more and more recognizable in modern data management, conformity assessment, and laboratory accreditation system because of its importance. In this paper, a set of reasonable probability explanations are introduced and an effective method is proposed to quantify the assessment indices for the uncertainty measurement of electrical testing laboratory. First of all, the influence from uncertainty factors during the test process is taken into account. With the use of ISO/IEC Guide 98-3 standard and probability theory, the index and model for the measurement uncertainty assessment of a laboratory is then derived. From the simulation results of safety testing, laboratory uncertainty measurement assessment activity for actual electrical appliances, and the confirmation of Monte Carlo simulation method, the appropriateness and correctness of proposed method are verified.
\end{abstract}

\section{Keywords}

Electrical Testing, Measurement Uncertainty, Testing Laboratory

\section{Introduction}

Because of global free trade, increasingly competitive markets, and rising consumer awareness, whether electrical test reports that are provided to numerous consumers by commercial electrical testing laboratories (performing electrical testing for high-voltage equipment, electromagnetic compatibility, and energy efficiency) are accurate and valid, satisfy market requirements, comply with laws and regulations, and are accepted by users are issues that have received extensive national and international attention. Testing is an activity during which an item or multiple items are measured by using specialized measurement equipment in the controlled environment of a laboratory; furthermore, testing is conducted by using qualitative and quantitative analyses to obtain a quantity of information about the characteristics of a research target [1]-[4].

Some evidence has shown that repeatedly measuring an identical quantity frequently yields inconsistent results. A difference between a measured value and its true value can result from incomplete measurement equipment, an unideal environment, and the limited ability of staff or methods. To adequately measure, accurately 
process measurement data, fully understand measurement-related issues, and avoid difficulties caused by using measurement results to determine whether products conform to requirements, the international community use uncertainty to explain the quality of measurement results. Small uncertainty indicates high quality, accuracy, and use value of measurement results; large uncertainty indicates low quality, accuracy, and use value of measurement results [5] [6]. According to the accreditation requirements for commercial testing laboratories in Taiwan and abroad, a commercial testing laboratory should develop procedures to measure and evaluate uncertainty to estimate the uncertainty of final test results and to avoid mistaking the reported results of uncertainty. Therefore, studies related to the evaluation, expression, and improvement of uncertainty in measurement are extremely crucial.

\section{Basic Concepts and Issues}

\subsection{Testing Laboratory}

Testing laboratories are required to undertake two main tasks. The first task is to coordinate and optimize the organizational framework, staff responsibilities, resource utilization, operation procedures, quality activities, and various types of information to form an organic measurement service system that is compatible with its service types, operation range, and workload. The second task is to ensure that reports on product testing (i.e. documents about test results or other related information) can satisfy customer requirements (e.g., the requirements of government departments, accreditation organizations) and enhance customers' confidence in product quality.

\subsection{Measurement}

This study used a mathematical function $Y_{i}$ (i.e., Equation (1)) to describe the information (i.e., measurement results) pertaining to a quantity that corresponds to a property of a measured object. The measurement results are typically represented by a single value and measurement errors, which are comparable, compatible, and traceable.

$$
Y_{i}=T_{i}+\phi+\alpha_{i}+\beta_{i}+\chi_{i}+\varepsilon_{i}
$$

where $Y_{i}$ denotes the obtained value for the $\mathrm{i}^{\text {th }}$ measurement, $T_{i}$ denotes the true value for the $\mathrm{i}^{\text {th }}$ measurement, $\phi$ denotes the bias of the measuring instrument, $\alpha_{i}$ denotes the influence of the measured object itself on the $\mathrm{i}^{\text {th }}$ measurement, $\beta_{j}$ denotes the influence of the measuring site on the $\mathrm{i}^{\text {th }}$ measurement, $\chi_{i}$ denotes the influence of staff's knowledge on the $\mathrm{i}^{\text {th }}$ measurement, and $\varepsilon_{i}$ denotes the random errors of the measuring instrument. To conveniently interpret, analyze, and ameliorate problems, the study used (2) to interpret (1):

$$
Y=f(x, w),
$$

where $x=\left[x_{1}, x_{2}, \cdots, x_{N}\right]$ denotes parameters of uncertainty sources that influence the accuracy of measurement results (e.g., staff's knowledge, measuring instrument, measurement procedure, and sample treatment) and $w$ denotes decision-making parameters, such as prevention or correction measures.

\subsection{Measurement Uncertainty}

Numerous studies have shown that measurement errors are inevitable and universal. Typically, errors are analyzed according to hypotheses. Because no one knows the true values, errors cannot be actually calculated and the term uncertainty is more appropriate than is the term error [7].

The meaning of studying uncertainty is as follows. First, using uncertainty rather than errors to represent measurement results can be easily understood and uncertainty can be easily estimated. Second, stipulating the format of the expression of measurement results can help users understand and accept measurement results.

\subsection{ISO/IEC Guide 98-3}

ISO/IEC Guide 98-3 is a crucial guide used worldwide to estimate and express uncertainty. Regarding the accreditation system for laboratories and inspection agencies, the Taiwan Accreditation Foundation-Chinese National Laboratory Accreditation-R06 (TAF-CNLA-R06) was formulated to request that testing laboratories and inspection agencies under accreditation evaluation refer to ISO/IEC Guide 98-3 for the evaluation and expres- 
sion of uncertainty in measurement [1]. The evaluation method adopted by ISO/IEC Guide 98-3 is shown in a cause and effect diagram in which factors influencing measurement results are quantified and then summed up to obtain the measurement uncertainty [8].

\subsection{The Influence of Measurement Uncertainty}

The conventional principle for conformity determination is that a measurement value $Y$ is compared with a product specification limit value (or specification limit value) $Y_{\text {lim }}$ to determine whether test results conform to requirements. However, the measurement value possesses a dispersion characteristic, and therefore the aforementioned conformity determination method cannot be used to determine conformity and a misjudgment can occur, as shown in Figure 1. In Figure 1, Conditions 1 and 4 do not require a consideration of influence from measurement uncertainty; however, for Conditions 2 and 3, whether measurement results conform to requirements may not be verifiable.

Assume that the allowable error range is $\pm T / 2$. According to the conventional principle for conformity determination, if a measurement value $Y$ falls in the allowable range $Y_{\lim }-T / 2 \leq Y \leq Y_{\lim }+T / 2$, then whether the measurement results conform to requirements can be determined. Similarly, the dispersion of the measurement value results in a problem related to the margin of conformity with requirements, as shown in Figure 2. In Figure 2, for Conditions 2 and 6, the probability of a qualified product is higher than is the probability of an unqualified product, and therefore the conclusion that the product is qualified can be drawn; however, a misjudgment can occur, thereby resulting in mistakenly rejecting a qualified product. For Conditions 3 and 7 , the probability of an unqualified product is higher than the probability of a qualified product, and therefore the conclusion that the product is unqualified can be drawn; however, a misjudgment can occur, thereby resulting in mistakenly accepting an unqualified product.

According to Figure 4 and Figure 5, determining whether measurement results conform to requirements must consider measurement uncertainty to avoid mistakenly rejecting qualified products or accepting unqualified products, violating regulations, causing trade disputes and unsafe consumption and usage, and affecting national and corporate economic interests because of substantial measurement uncertainty or inadequate conformity determination methods. This is the meaning of the evaluation, expression, and improvement of uncertainty in measurement.

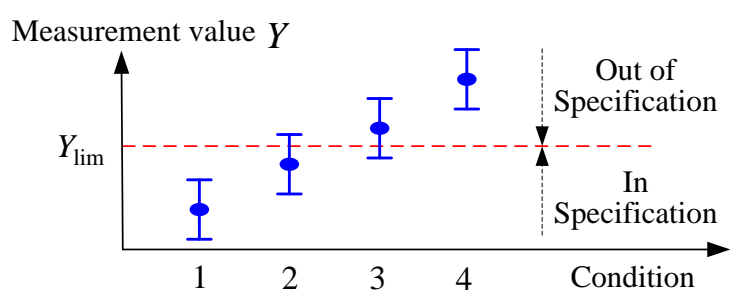

Figure 1. Conformity relationship between measurement uncertainty and measurement results.

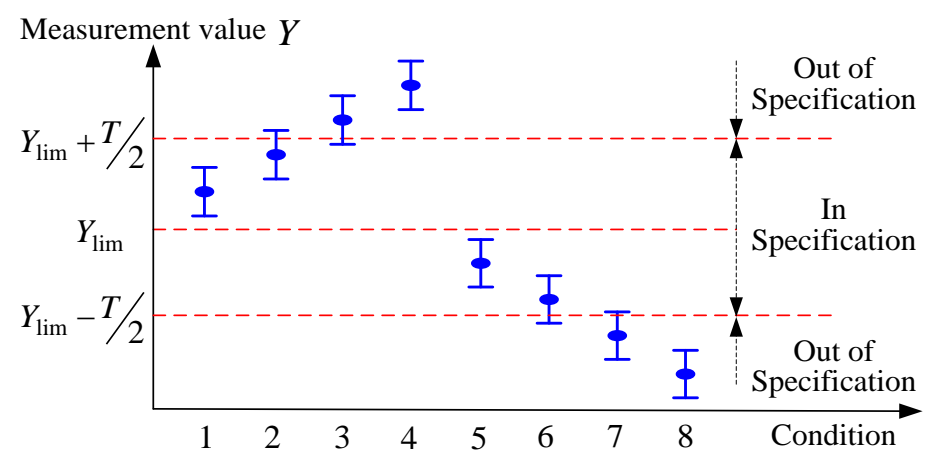

Figure 2. The relationship between the dispersion of measurement results and conformity determination. 


\section{Probabilistic Approach of the Measurement Uncertainty Evaluation}

The accuracy and reliability of measurement results obtained from testing operations in a laboratory are determined by numerous factors; thus, the adequacy of measurement processes and the accuracy and reliability of measurement results must be represented by uncertainty, which serves as a basis for tracing and controlling measurement processes to ensure that measurement results conform to requirements. Based on this, this study proposed a method for the evaluation, expression, and improvement of uncertainty in electrical measurement. The method is elaborated as follows [9]-[12].

\subsection{Describing and Calculating the Measurement Quantification}

Assume that for a testing operation, the measurement result $Y$ refers to a function $Y=f\left(x_{1}, x_{2}, \cdots, x_{N}\right)$ containing $N$ other measured amounts $x$, and that its probability density function is $f_{x}(x)$. The function $Y=f(x)$, where $x$ is uncertainty parameter, can be expanded by using statistical linear approximation based on the expected value of $x$ (i.e. $\mu_{x}$ ), as expressed by (3).

$$
y=f\left(\mu_{x_{1}}, \mu_{x_{2}}, \cdots, \mu_{x_{n}}\right)+\sum_{i=1}^{N}\left(x_{i}-\mu_{x_{i}}\right) \frac{\mathrm{d} y}{\mathrm{~d} x_{i}}+\frac{1}{2} \sum_{i=1}^{N} \sum_{\substack{j=1 \\ i \neq j}}^{N}\left(x_{i}-\mu_{x_{i}}\right)\left(x_{j}-\mu_{x_{j}}\right) \frac{\mathrm{d}^{2} y}{\mathrm{~d} x_{i} \mathrm{~d} x_{j}}+\cdots
$$

By removing all items after the linear term in (3), the approximate values of the expected value $E(y)$ and the variance $\operatorname{Var}(y)$ of $y=f\left(x_{1}, x_{2}, \cdots, x_{N}\right)$ can be obtained as follows:

$$
E(y) \approx f\left(\mu_{x_{1}}, \mu_{x_{2}}, \cdots, \mu_{x_{n}}\right)
$$

and

$$
\operatorname{Var}(y) \approx \sum_{i=1}^{N} \operatorname{Var}\left(x_{i}\right)\left(\left.\frac{\partial f}{\partial x_{i}}\right|_{x_{i}=\mu_{x_{i}}}\right)^{2}+2 \sum_{i=1}^{N-1} \sum_{j=i+1}^{N} \operatorname{Cov}\left(x_{i}, x_{j}\right)\left(\left.\frac{\partial f}{\partial x_{i}}\right|_{x_{i}=\mu_{x_{i}}}\right)\left(\left.\frac{\partial f}{\partial x_{j}}\right|_{x_{j}=\mu_{x_{j}}}\right)
$$

where $x_{i}$ and $x_{j}$ (within $\left[x_{1}, x_{2}, \cdots, x_{N}\right]$ ) denote the parameters of measurement uncertainty sources, $\operatorname{Cov}\left(x_{i}, x_{j}\right)$ denotes the covariance of $x_{i}$ and $x_{j}$, equaling the product of standard deviation $\sigma\left(x_{i}\right)=\sqrt{\operatorname{Var}\left(x_{i}\right)}$, standard deviation $\sigma\left(x_{j}\right)=\sqrt{\operatorname{Var}\left(x_{j}\right)}$, and correlation coefficient $\rho\left(x_{i}, x_{j}\right)$. If $x_{i}$ and $x_{j}$ are independent of each other (i.e., $\rho\left(x_{i}, x_{j}\right)=0$ ), then (6) can be used to calculate the uncertainty of measurement result $Y$.

$$
\operatorname{Var}(y) \approx \sum_{i=1}^{N} \operatorname{Var}\left(x_{i}\right)\left(\left.\frac{\partial f}{\partial x_{i}}\right|_{x_{i}=\mu_{x_{i}}}\right)^{2}
$$

\subsection{Describing and Calculating the Dispersion of Measurement Results}

Equations (5) and (6) can be widely applied to express the uncertainty of measurement results. Measurement results are expressed using the formula $Y=y \pm u_{c}$ (the probability of the true value of a measured event is only $68 \%$ ). For commercial, industry, environmental, safety, and hygienic applications, measuring the dispersion of measurement results is often required to estimate the rational range of measurement results and to evaluate whether uncertainty influences conformity determination (i.e., whether uncertainty exceeds specification limits). The dispersion of measurement results is called measurement uncertainty.

In this study, to easily interpret, analyze, and improve the dispersion and range of measurement results, the measurement results function $Y=f\left(x_{1}, x_{2}, \cdots, x_{N}\right)$ is converted into a confidence interval that corresponds to a probability level $\alpha_{L}$ to evaluate the dispersion of measurement results, as shown in Figure 3. Accordingly, considering uncertainty (or dispersion), measurement results $Y$ can be expressed as follows:

$$
Y=E(y) \pm \phi^{-1}\left(\alpha_{L}\right) \sqrt{\operatorname{Var}(y)}
$$

where $E(y)$ and $\operatorname{Var}(y)$ denote the estimated mean and standard deviation of $f\left(x_{1}, x_{2}, \cdots, x_{N}\right), \phi^{-1}\left(\alpha_{L}\right)$ 


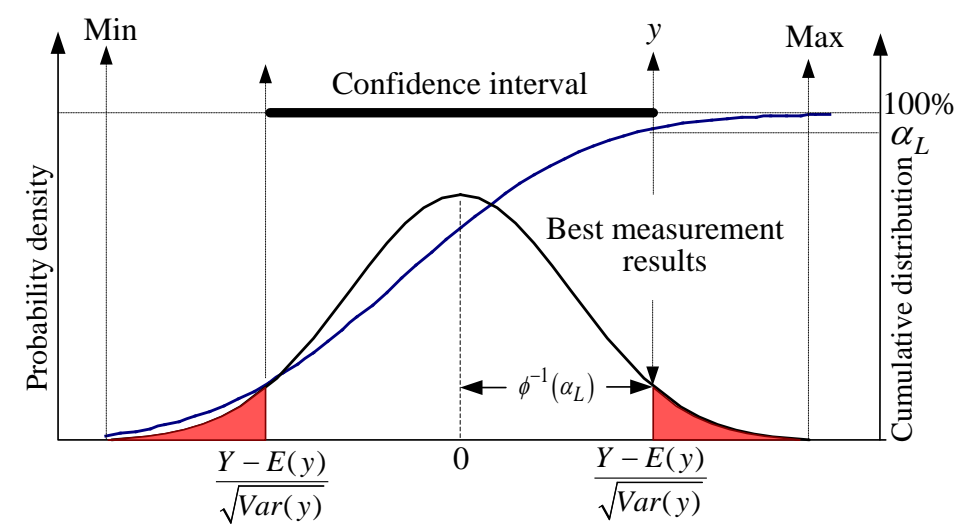

Figure 3. The confidence interval of measurement results.

denotes the value of the inverse normal cumulative probability function of a probability level $\alpha_{L}$ (according to the table, $\left.\phi^{-1}(95 \%)=1.96\right)$, and $\phi^{-1}\left(\alpha_{L}\right) \sqrt{\operatorname{Var}(y)}$ is the estimated value of measurement uncertainty.

\subsection{The Expression of Test Results That Consider Measurement Uncertainty}

Two methods for expressing test results are numerical and nonnumerical methods. Regarding the numerical method, when technical standards or customers require using a numerical method or uncertainty affects conformity determination, the expression of test results must consider measurement uncertainty to ensure the validity and accuracy of test results, as expressed by (8).

$$
\begin{gathered}
{ }^{1} U_{95 \%}=\left(\begin{array}{c}
14.5 \\
2 .
\end{array}{ }_{3.5}\right) V^{4 .} \\
\begin{array}{c}
\text { The end } \\
\text { result }
\end{array}=\begin{array}{c}
\text { Measurement } \\
\text { value }
\end{array} \text { (Average value) } \pm \text { measurement } \\
\begin{array}{c}
\text { 1.Symbol 2.Numerical value of measured quantity } \\
\text { interval 95\%) }
\end{array} \\
\begin{array}{c}
\text { 4.Unit } \\
\text { uncertainty(Confidence }
\end{array}
\end{gathered}
$$

Regarding the nonnumerical method (e.g., passing or failing a test), if a testing method has clearly defined technical standards, customer requirements, and operation regulations, then it can be assumed that the testing method has considered measurement uncertainty and therefore estimating uncertainty is not required (i.e., a special case of risk sharing). The difference between the method and other methods and the degree to which the validity of the measurement results is affected must be evaluated and reported.

\subsection{Amelioration of the Measurement Uncertainty Problem}

As shown in Figure 4, regarding the measurement results $(Y)$ for a designated test item in a laboratory, a large measurement uncertainty $U$ may lead to a misjudgment about whether products conform to requirements, thereby resulting in mistakenly rejecting or accepting products. To ameliorate the problem related to measurement uncertainty, a laboratory must take preventive and corrective measures to reduce the influence of measurement uncertainty $U$ and ensure that measurement uncertainty falls within an acceptable range $Y_{\lim }$ (i.e., $\left.U \leq U^{*}\right) . U^{*}$ denotes the optimal value of measurement uncertainty, as expressed by (9).

$$
U^{*}=\phi^{-1} \sqrt{\operatorname{Var}(y)^{*}}
$$

where

$$
\operatorname{Var}(y)^{*} \approx \sum_{i=1}^{N} \operatorname{Var}\left(x_{i}\right)\left(\left.\frac{\partial f^{*}}{\partial x_{i}}\right|_{x_{i}=\mu_{x_{i}}}\right)^{2}+2 \sum_{i=1}^{N-1} \sum_{j=i+1}^{N} \operatorname{Cov}\left(x_{i}, x_{j}\right)\left(\left.\frac{\partial f^{*}}{\partial x_{i}}\right|_{x_{i}=\mu_{x_{i}}}\right)\left(\left.\frac{\partial f^{*}}{\partial x_{j}}\right|_{x_{j}=\mu_{x_{j}}}\right)
$$

In (10), $f^{*}=f(x, w)$, where $x=\left[x_{1}, x_{2}, \cdots, x_{N}\right]$, denotes the uncertainty source parameters of measurement results and $w=\left[w_{1}, w_{2}, \cdots, w_{N}\right]$ denotes the decision-making parameters for preventive and corrective measures. 


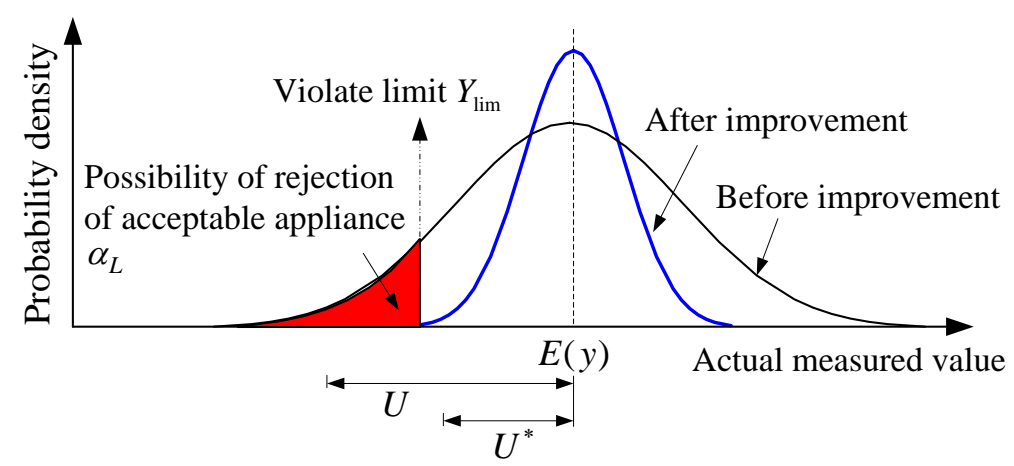

Figure 4. Amelioration of the influence of uncertainty in measurement results.

Such measures include establishing and implementing an adequate equipment management system, ensuring the measurement traceability and accuracy of measurement equipment, maintaining the quality of measurement environments, and enhancing professional skills and knowledge among people who conduct measurements.

This study used the measurement traceability of instruments and equipment as an example to explain preventive and corrective measures. Measurement traceability means that because a reading obtained from a measuring instrument contains measurement errors and uncertainty $(e)$, in one or multiple stages, standard devices or samples are used to calibrate measurement equipment and identify the relationship between a reading and true value and then a physical method is used to adjust and correct the measurement equipment ( $k$ denotes the correction procedure). Thus, the reading obtained from the measurement equipment can be compared with national or international standard values. Through such a sequence of comparisons, the measurement results $x_{0}$ are converted into the standard value $x_{0}^{\prime}$ based on the International System of Units (SI).

\subsection{Enunciating the Method Implementation}

The steps for implementing the method proposed in this study for the evaluation, expression, and improvement of uncertainty in electrical measurement are elaborated as follows:

Step 1 Determine the measurement result equation (i.e., $y=f\left(x_{1}, x_{2}, \cdots, x_{N}\right)$ );

Step 2 Determine the uncertainty source parameters (i.e., $x_{1}, x_{2}, \cdots, x_{N}$ ) that influence measurement results $y$. Uncertainty sources include staff, measuring instruments, environmental conditions, operation methods, and sample treatment;

Step 3 Identify the descriptive parameters of a probability distribution, that is the expected value and standard deviation, of uncertainty source parameters (i.e., $x_{1}, x_{2}, \cdots, x_{N}$ ) according to sampling theory and probability distribution;

Step 4 Use the expected value and standard deviation obtained in Step 3 and combine them with (5) and (6) to calculate the probability parameters of the expected value $E(y)$ and variance $\operatorname{Var}(y)$ of $y=f\left(x_{1}, x_{2}, \cdots, x_{N}\right)$;

Step 5 Substitute the expected value and variance obtained in Step 4 into (7) to calculate the measurement results with a probability level $\alpha_{L}$;

Step 6 After evaluating the measurement results and expressing the related uncertainty according to (8), present the results in a test report. Stop and conclude the procedure.

Step 7 If a misjudgment about whether products conform to requirements occurs because of substantial measurement uncertainty, then propose preventive and corrective measures testing staff, measurement equipment, environmental conditions, and measurement methods according to (9). Then return to Step 1.

\section{Application Case}

This study used the actual operations performed by a commercial laboratory for electrical appliances safety testing as an example to explain the feasibility of the method for the evaluation, expression, and improvement of uncertainty in measurement [13]. The test sample is electric instant water heater, the main specification is AC $220 \mathrm{~V} 60 \mathrm{~Hz} 8.8 \mathrm{~kW}$. The power consumption of the test sample was measured. The measurement method implemented is based on Section 10.1 in the Chinese National Standards IEC 60335-1 and IEC 60335-2-59. 


\subsection{Calculating the Uncertainty of Measurement Results}

Assume that the measurement results for the power consumption of the electric instant water heater can be expressed by the equation $P=E I \operatorname{Cos} \theta$ and identify the uncertainty sources that influence the measurement results. Table 1 shows the statistics for the standard deviation or variance of each quantified uncertainty source. The correlation coefficient $\operatorname{Cov}\left(x_{i}, x_{j}\right)$ is 0.5 . According to the specification limits for the power consumption of the product based on IEC 60335-1 and IEC 60335-2-59, the allowable difference between the power consumption of the product and the labeled rated power consumption cannot exceed the range of $-10 \%$ to $+5 \%$.

According to the calculation results obtained from applying (5) to (7), the measurement value for the power consumption of the electric instant water heater is $8998 \mathrm{~W}$ and the measurement uncertainty with a $95 \%$ confidence interval is $1942 \mathrm{~W}$. According to (8), the measurement results can be expressed as $P_{95 \%}=8998 \pm 1942 \mathrm{~W}$. Subsequently, whether the measurement results for power consumption met the standards stipulated in IEC 60335-1 and IEC $60335-2-59$ and were within the allowable range of $-10 \%$ to $+5 \%$ was determined. According to the conventional principle for conformity determination, the measurement value for power consumption fell within the allowable range (i.e., $7920 \mathrm{~W} \leq 8998 \mathrm{~W} \leq 9680 \mathrm{~W}$ ) and therefore the test results conformed to requirements. However, considering the dispersion of measurement results, the measurement results for power consumption exceed the upper limit of specifications and therefore a misjudgment may occur, as shown in Figure 5. Accordingly, qualified products may be mistakenly rejected, thereby resulting in demand for legal regulation and trade disputes.

\subsection{Reducing the Influence of Measurement Uncertainty}

If it cannot be determined whether the aforementioned power consumption measurement value conforms to the requirements within a particular confidence interval, the concepts and methods provided in Section 3.5 in this paper can be used to identify factors that significantly influence the dispersion of the measurement value and therefore related measures can be taken to improve or eliminate the factors (Figure 6), to reduce the influence of measurement uncertainty, and to enhance the quality of measurement processes and results.

\subsection{Comparing and Verifying the Proposed Method}

Finally, this study used the Monte Carlo simulation to simulate and verify the proposed method for the evaluation,

Table 1. Variability in the sample testing process $(\sigma \%)$.

\begin{tabular}{|c|c|c|c|}
\hline Source of variation in testing process & Probability distribution & $\mu\left(x_{i}\right)$ & $\sigma\left(x_{i}\right)$ \\
\hline Power supply $\mathrm{x}_{1}$ & Normal & 220 & 2.8 \\
\hline Load current $\mathrm{x}_{2}$ & Normal & 40.9 & 1.4 \\
\hline Repeatability of voltage regulator $x_{3}$ & Rectangular & - & 4.3 \\
\hline Repeatability of voltage regulator $\mathrm{x}_{4}$ & Rectangular & - & 6.7 \\
\hline Reliability of test staff $\mathrm{x}_{5}$ & Normal & - & 5.8 \\
\hline Traceability of power meter calibration $\mathrm{x}_{7}$ & - & - & 3.8 \\
\hline
\end{tabular}

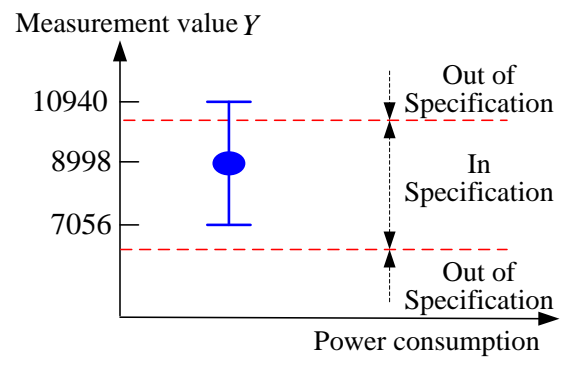

Figure 5. Power consumption measurement results. 


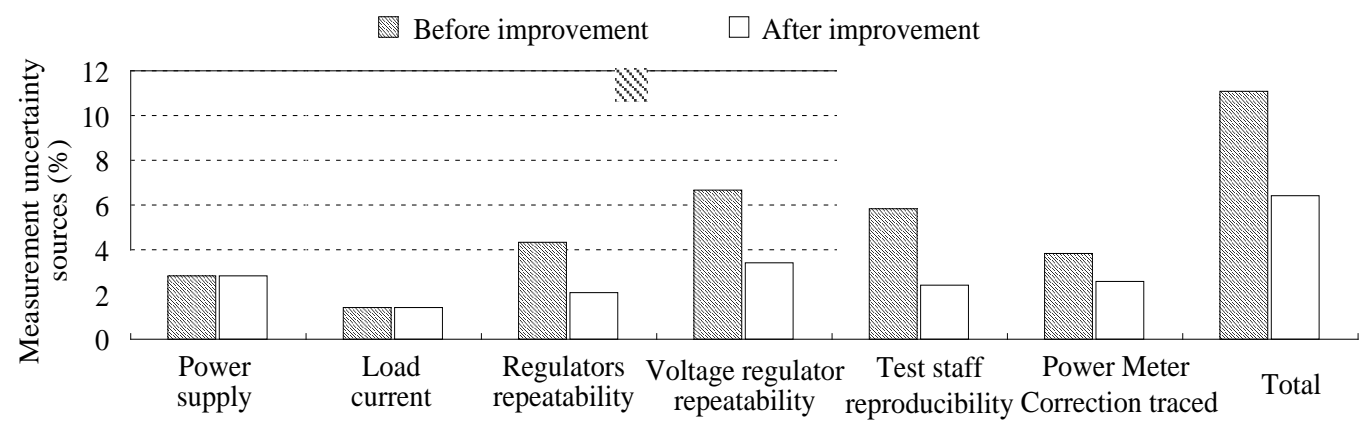

Figure 6. Strategies for reducing influence from measurement uncertainty.

expression, and improvement of uncertainty in measurement [14] [15]. The Monte Carlo simulation reflects the randomness and uncertainty of an event and, thus, is an efficient and easily implemented simulation method. To simulate the power consumption measurement shown in Table 1, 1000 simulative iterations were performed to obtain adequate simulation results. The simulated expected value and variance differed from the estimated expected value and variance obtained using the method proposed in this study by only $3 \%$ and $5 \%$, respectively. This demonstrated the similarity of the results obtained through the proposed method and the Monte Carlo simulation.

\section{Conclusions}

In the field of electrical testing, using test results that consider measurement uncertainty is extremely crucial and can avoid the mistaken rejection of qualified products and the acceptance of unqualified products, thereby preventing regulatory violations, trade disputes, and unsafe consumption and use.

Measurement uncertainty is used to rationally characterize the dispersion of a measurement value and parameters related to measurement results, helping ensure the accuracy and reliability of measurement results and the accuracy of judgment regarding whether products conform to requirements, assisting in the acquisition of laboratory accreditation. In response to actual requirements, this study used probability theory, a Monte Carlo simulation method, ISO/IEC 17025, and ISO/IEC Guide 98-3 to establish and verify a probability analytical model and evaluation method in order to evaluate, express, and improve uncertainty in electrical measurement. This study also considered uncertainty sources such as measurement staff, measurement devices, environmental conditions, operation methods, and sample treatment when quantifying measurement operations, estimated statistics for the dispersion of measurement results, explained the expression of test results, and described the method for improving and solving problems. Accordingly, the reliability of measurement results can be controlled and the effective measures to regulate testing activities can be taken.

The method proposed in this study can be applied to product development, food and drugs, environmental protection, lawsuits, product accreditation, factory quality control, insurance claims, sales, regulatory requirements, forensic science, and other testing-related problems.

\section{References}

[1] Taiwan Accreditation Foundation. Data Area Laboratory Accreditation. http://www.taftw.org.tw/

[2] Bureau of Standards, Metrology \& Inspection, M.O.E.A. Commodity Inspection Plan Designated Laboratory Certification Services. http://www.bsmi.gov.tw/

[3] Bureau of Energy, Ministry of Economic Affairs. Energy Efficiency Testing Laboratory Certification Program. http://www.energylabel.org.tw/

[4] National Communications Commission. Information Technology Security Testing Laboratory Accreditation Service Plan. http://www.ncc.gov.tw/

[5] ISO 10012 (2003) Measurement Management Systems-Requirements for Measurement Processes and Measuring Equipment.

[6] ISO/IEC17025 (2005) General Requirements for the Competence of Testing and Calibration Laboratories.

[7] ISO/IEC Guide 98-3 (2008) Uncertainty of Measurement-Part 3, Guide to the Expression of Uncertainty in Measurement. 
[8] Wang, Z., Liu, Z., Xia, T. and Zhulian, Q. (2008) Uncertainty Evaluation and Measurement Error. Science Press.

[9] Ridler, N., Lee, B., Martens, J. and Wong, K. (2007) Measurement Uncertainty, Traceavility, and the GUM. IEEE Microwave Magazine on Microwave Magazine, 8, 44-53. http://dx.doi.org/10.1109/MMW.2007.383952

[10] Giordani, A. and Mari, L. (2012) Measurement, Models, and Uncertainty. IEEE Transactions on Instrumentation and Measurement, 61, 2144-2152. http://dx.doi.org/10.1109/TIM.2012.2193695

[11] Leon-Garcia, A. (1994) Probability and Random Processes for Electrical Engineering. Addison-Wesley.

[12] Chang, G.W., Wang, H.L. and Chu, S.Y. (2007) A Probabilistic Approach for Optimal Passive Harmonic Filter Planning. IEEE Transactions on Power Delivery, 22, 1790-1798. http://dx.doi.org/10.1109/TPWRD.2007.899230

[13] Wang, H.L. (2006) Research and Test Activities Interval Estimation Method Applied in the Field of Electrical Capacity. The 2006 Conformity Assessment and Certification Seminar, May.

[14] Rubinstein, R.Y. (1981) Simulation and the Monte Carlo Method. Addison-Wesley. http://dx.doi.org/10.1002/9780470316511

[15] Leinhos, J. and Arz, U. (2008) Monte-Carlo Analysis of Measurement Uncertainties for On-Wafer Thru-Reflect-Line Calibrations. IEEE Transactions on Microwave Symposium Digest, 22, 33-36.

http://dx.doi.org/10.1109/MWSYM.2008.4633327 